\title{
Analysis of the Free Vibration of a Coupled Plate/Fluid Interacting System and Interpretation using Sub-system Modal Energy
}

\author{
Daniel G Gorman ${ }^{1}$ \\ Department of Mechanical Engineering, James Weir Building, \\ University of Strathclyde, Glasgow G1 1XJ
}

Jaromír Horáček

Institute of Thermomechanics, Academy of Sciences of the Czech Republic, Dolejškova 5,182 00 Prague 8, Czech Republic

\begin{abstract}
This paper describes a method for describing and quantifying the vibratory behaviour of interacting structural/fluid systems based upon reference to the relative energy associated with each of the sub-systems. The particular case selected is that of a circular plate in interaction with a cylindrical fluid cavity. A theoretical analysis is performed, based upon the Euler Bernoulli and Helmhotz equations combined through a Galerkin technique, from which the natural frequencies and associated mode functions of the interacting system are calculated. The convergence of the analysis is investigated and the opportunity is taken to investigate the sensitivity of the coupled natural frequencies to different assumed mode shapes of the plate in vacuo. Subsequently the coupled mode functions are used to describe details of the energy associated with the plate and the fluid. It is found that presentation of these relative energies renders a satisfactory insight into the vibration behaviour of the coupled system.
\end{abstract}

Keywords Vibrations, structural/fluid interaction, structural/acoustic.

\section{INTRODUCTION}

The influence of fluid interaction upon the higher frequency vibration of a light flexible structure has been a subject of growing interest, particularly due to the increased deployment of thin-walled liquid/gas containers such as pipes and storage vessels. In the literature this work comes under two main headings; structural/acoustic vibration interaction and structural/fluid vibration interaction. In the former the research is confined to the interaction between a light structure and sound pressure waves in an acoustic cavity which the structure is enclosing. The general analysis of acoustic/structural vibration interaction problems is presented in $[1,2]$, where infinite series solutions for the acoustic pressure and the displacement of 
the structure are derived from a fundamental solution of the uncoupled problems, viz.: vibration of the structure in vacuo, and acoustic resonance in a closed cavity with undeformable walls. These basic models were extended and applied to problems involving rectangular plates backed by rectangular cavities $[3,4,5,6]$. A study has been performed on the case of a circular membrane vibrating in contact with a gas contained in both a closed and open cylindrical cavity [7]. With respect to the case of vibro-acoustic effects involving a circular plate, Lee and Singh [8] analysed the characteristics of the acoustic radiation emitted from a vibrating circular plate in free space and Gorman et al considered the case of a circular disc covering a cylindrical acoustic cavity in the absence of [9] and presence [10] of inplane membrane stress in which they developed coupled system equations for iterating the coupled natural frequencies only. With respect to structural/fluid vibration interaction in storage containers, Bauer and Chiba [11] considered the case of a circular plate backed by a cylindrical cavity containing fluid assumed to be viscous and incompressible and Amabili considered effect of incompressible liquid depth and contact upon the free vibration of circular and annular plates [12,13] . Recently Gorman et al [14] studied the case of a circular plate in interaction with a liquid/gas cavity which included the dimension of the interaction between the liquid and gas filled cavities.

In studies such as this, since in all cases one is dealing with some degree of structural/fluid vibration interaction, it would be erroneous to describe any mode of vibration as either purely a structural mode or an acoustic (fluid) mode. Rather the natural modes associated with such interacting systems are interacting modes of the structure and the fluid and the question is posed; for any interacting mode of vibration, what is it structurally or fluid dominant and to what relative degree? Accordingly, the main aim of this paper is to develop and describe a method, based upon sub-system modal energy, which will quantifying and describing the natural modes of vibration of the plate/fluid interacting system with respect to the relative structural or fluid dominance. Prior to this, the analysis will be described and the convergence of the method for predicting natural frequencies and associated mode shapes investigated.

\footnotetext{
${ }^{1}$ Corresponding author. Department of Mechanical Engineering, University of Strathclyde, Glasgow G1 1XJ, Scotland, UK, Tel +44 1415482210, Fax +44 141 552 5105, Email Daniel.gorman@strath.ac.uk.
} 


\section{BASIC ANALYSIS OF THE VIBRATION OF THE COUPLED SYSTEM.}

The equation of motion, describing the free small axisymmetric lateral vibration, $w=$ $w(r, t)$, of a circular disc in interaction with the acoustic cavity, as shown in Figure 1, is

$$
\nabla^{4} \bar{w}=\frac{-\rho_{d} h a^{4}}{D} \frac{\partial^{2} \bar{w}}{\partial t^{2}}+\frac{p a^{3}}{D}
$$

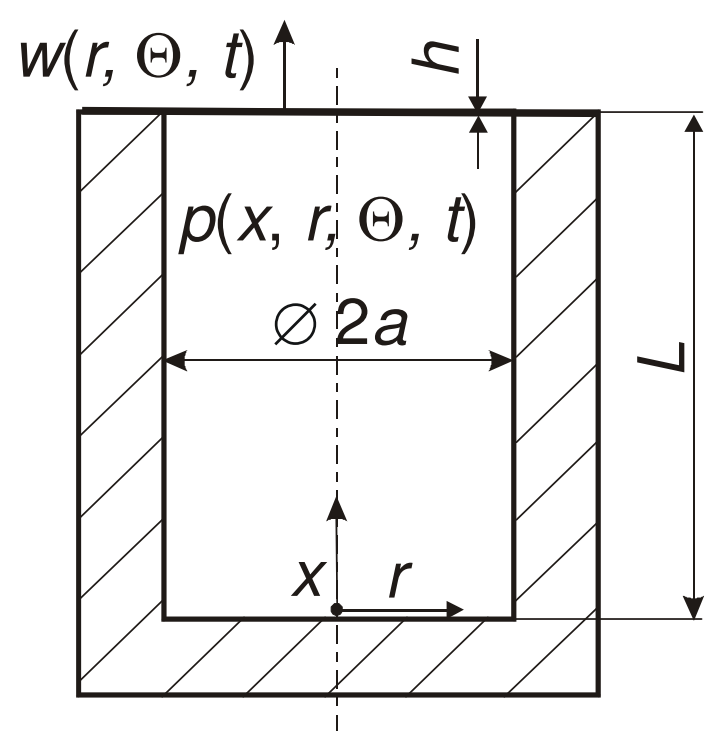

Figure 1 - Schematic diagram

Also for axisymetric modes of vibration,

$$
\nabla^{2}=\left(\frac{\partial^{2}}{\partial \bar{r}^{2}}+\frac{1}{\bar{r}} \frac{\partial}{\partial \bar{r}}\right), \bar{w}=w / a, \bar{r}=r / a \text { and } D=E h^{3} / 12\left(1-\mu^{2}\right) ;
$$

$E$ is Young's modulus, $\mu$ is Poisson ratio and $\rho_{d}$ is the plate density; $a$ and $h$ are the radius and thickness of the plate, respectively; $L$ is the length of the cylindrical cavity and $p$ is the acoustic pressure inside.

Now writing

$$
\bar{w}=\sum_{s=1}^{\infty} \chi_{s} \psi_{s}(\bar{r}) e^{i \omega t}
$$


where $\psi_{s}(\bar{r})$ is the natural mode shape of the disc in vacuo and $\chi_{s}$ is a constant for that mode, generally referred to as the mode shape coefficient for the mode consisting $s$ nodal circles. In this particular case, for a stressed disc clamped at the periphery, the mode shapes, $\psi_{S}(\bar{r})$, are according to [15]:

$$
\psi_{s}(\bar{r})=\frac{-I_{0}\left(\xi_{s}\right)}{J_{0}\left(\xi_{s}\right)} J_{0}\left(\xi_{s} \bar{r}\right)+I_{0}\left(\xi_{s} \bar{r}\right)
$$

where $\xi_{s}$ are roots (values of $s=1,2,3$ etc.) computed from the equation:

$$
0=\frac{J_{1}\left(\xi_{s}\right)}{J_{0}\left(\xi_{s}\right)}+\frac{I_{1}\left(\xi_{s}\right)}{I_{0}\left(\xi_{s}\right)}
$$

Where $I_{0}, I_{1}$ and $J_{0}, J_{1}$ are the Bessel functions (order zero and one).

For a particular value of $s$, the natural frequency of free undamped vibration, $\omega_{s}$, is then:

$$
\omega_{s}=\xi_{s}^{2} \sqrt{\frac{D}{\rho_{d} h a^{4}}} .
$$

Now for a particular mode of vibration for the disc in vacuo:

$$
\nabla^{4}\left[\psi_{s}(\bar{r})\right]=\frac{\omega_{s}^{2} \rho_{d} h a^{4}}{D} \psi_{s}(\bar{r}) .
$$

Therefore combination of equations (1), (2) and (6) gives:

$$
\sum_{s=1}^{\infty}\left[\left(\omega_{s}^{2}-\omega^{2}\right) \chi_{s} \psi_{s}(\bar{r})\right] e^{i \omega t}=\frac{p}{\rho_{d} h a} .
$$

The form of the acoustic pressure, $p$, acting on the disc will now be established by reference to the acoustic cavity. Consider the acoustic cavity shown in Figure 1, whose velocity potential, $\phi=\phi(x, r, t)$ is described by

$$
\frac{\partial^{2} \bar{\phi}}{\partial \bar{r}^{2}}+\frac{1}{\bar{r}} \frac{\partial \bar{\phi}}{\partial \bar{r}}+\left(\frac{a}{L}\right)^{2} \frac{\partial^{2} \bar{\phi}}{\partial \bar{x}^{2}}=\left(\frac{a}{c}\right)^{2} \frac{\partial^{2} \bar{\phi}}{\partial t^{2}},
$$

where $\bar{\phi}=\phi / a c, \quad \bar{x}=x / L$ and $c$ is speed of sound. Now writing

$$
\bar{\phi}=H(\bar{x}) \cdot Q(\bar{r}) e^{i \omega t}
$$

and substituting equation (10) into (9) gives

$$
\left(\frac{a}{L}\right)^{2} \frac{H^{\prime \prime}}{H}=-\left[\frac{Q^{\prime \prime}}{Q}+\frac{1}{\bar{r}} \frac{Q^{\prime}}{Q}+\lambda^{2}\right]= \pm k^{2},
$$

where $\lambda=\frac{\omega a}{c}$ and $k$ is a constant. For the right hand side of equation (10) equal to $-k^{2}$ gives

$$
Q(\bar{r})=B J_{0}(\alpha \bar{r})+\widetilde{B} Y_{0}(\alpha \bar{r})
$$


where $\alpha=\sqrt{\lambda^{2}-k^{2}}$ or $k=\sqrt{\lambda^{2}-\alpha^{2}}$ and $\widetilde{B}=0$ since $Q(\bar{r})$ must be finite when $\bar{r} \rightarrow 0$. At $\bar{r}=1$

$$
\frac{\partial \bar{\phi}}{\partial \bar{r}} \equiv \frac{d Q}{d \bar{r}}=0
$$

Therefore the condition (11) has roots $\alpha_{q}(q=1,2,3$ etc.), which satisfy the equation $J_{0}^{\prime}(\alpha)=0$.

Similarly $H=C \cos \left(\gamma_{q}^{(\omega)} \bar{x}\right) \quad$ since $\left.\frac{d H}{d \bar{x}}\right|_{\bar{x}=0}=0$,

where $\quad \gamma_{q}^{(\omega)}=\frac{L}{a} \sqrt{\lambda^{2}-\alpha_{q}^{2}}=\frac{L}{a} k_{q}^{(\omega)}$.

Therefore equation (9) becomes:

$$
\bar{\phi}=\sum_{q=1}^{\infty} B_{q} \cos \left(\gamma_{q}^{(\omega)} \bar{x}\right) J_{0}\left(\alpha_{q} \bar{r}\right) e^{i \omega t} .
$$

At $\bar{x}=1$, the axial component of the velocity of the gas and the lateral velocity of the plate must be equal, i.e,

$$
\left.\frac{c}{L} \frac{\partial \bar{\phi}}{\partial \bar{x}}\right|_{\bar{x}=1}=\frac{\partial \bar{w}}{\partial t} \quad \text { for } 0 \leq \bar{r} \leq 1 .
$$

Therefore combining equations (2) and (12) renders

$$
-\frac{c}{L} \sum_{q=1}^{\infty}\left[B_{q} \gamma_{q}^{(\omega)} \sin \left(\gamma_{q}^{(\omega)}\right) J_{0}\left(\alpha_{q} \bar{r}\right)\right]=i \omega \sum_{s=1}^{\infty} \chi_{s} \psi_{s}(\bar{r}) .
$$

Now using the orthogonal properties of the eigenfunction, $\bar{r} J_{0}\left(\alpha_{q} \bar{r}\right)$, by multiplying both sides of equation (13) by $\bar{r} J_{0}\left(\alpha_{q} \bar{r}\right)$ and integrating between $0 \leq \bar{r} \leq 1$ according to reference [16] gives

$$
B_{q}=\frac{-2 i \omega L}{c} \frac{\sum_{s=1}^{\infty} \chi_{s} K_{q s}}{\gamma_{q}^{(\omega)} \sin \left(\gamma_{q}^{(\omega)}\right) J_{0}^{2}\left(\alpha_{q}\right)},
$$

where $K_{q S}=\int_{0}^{1} \bar{r} \psi_{S}(\bar{r}) J_{0}\left(\alpha_{q} \bar{r}\right) d \bar{r}$

the value of which can be obtained through standard numerical integration.

Now the pressure, $p$, at the surface of the plate is given by:

$$
p=-\left.\rho_{f} a c \frac{\partial \bar{\phi}}{\partial t}\right|_{\bar{x}=1},
$$

where $\rho_{f}$ is the fluid density.

Therefore combining equations (12) and (14) renders: 


$$
p=-2 \omega^{2} a L \rho_{f} \sum_{s=1}^{\infty} \sum_{q=1}^{\infty} \frac{\chi_{s} K_{q s} J_{0}\left(\alpha_{q} \bar{r}\right)}{\left(\gamma_{q}^{(\omega)} \tan \gamma_{q}^{(\omega)}\right) J_{0}^{2}\left(\alpha_{q}\right)} e^{i \omega t}
$$

Substituting equation (16) into equation (7) gives:

$$
\sum_{s=1}^{\infty}\left(\omega_{s}^{2}-\omega^{2}\right) \chi_{s} \psi_{s}(\bar{r})=-2 \omega^{2} \frac{\rho_{f} L}{\rho_{d} h} \sum_{s=1}^{\infty} \sum_{q=1}^{\infty} \frac{\chi_{s} K_{q s} J_{0}\left(\alpha_{q} \bar{r}\right)}{\left(\gamma_{q}^{(\omega)} \tan \gamma_{q}^{(\omega)}\right) J_{0}^{2}\left(\alpha_{q}\right)} .
$$

Multiplying both sides by $\bar{r} J_{0}\left(\alpha_{q} \bar{r}\right)$ and integrating between $0 \leq \bar{r} \leq 1$ renders:

$$
\sum_{s=1}^{\infty} \chi_{s} K_{q s}\left\{\omega_{s}^{2}-\omega^{2}\left[1-\frac{\rho}{\left(\gamma_{q}^{(\omega)} \tan \gamma_{q}^{(\omega)}\right)}\right]\right\}=0,
$$

where $\rho=\frac{\rho_{f} L}{\rho_{d} h}=\frac{\text { mass of gas }}{\text { mass of plate }}$.

Now, since $\quad \omega_{s}^{2}=\xi_{s}^{4} \frac{D}{\rho_{d} h a^{4}}$

a quantity $\xi$ instead of $\omega$ can be introduced defined by the relation:

$$
\omega^{2}=\xi^{4} \frac{D}{\rho_{d} h a^{4}} \text {. }
$$

Hence equation (17) can be re-written as

$$
\sum_{s=1}^{\infty} \chi_{s} K_{q s}\left\{\xi_{s}^{4}-\xi^{4}\left[1-\frac{\rho}{\left(\gamma_{q}^{(\xi)} \tan \gamma_{q}^{(\xi)}\right)}\right]\right\}=0,
$$

where $\gamma_{q}^{(\xi)}=\frac{L}{a} \sqrt{\xi^{4} \frac{D}{\rho_{d} h a^{2} c^{2}}-\alpha_{q}^{2}}$.

Equation (19) can be represented in matrix form as

$$
\left[\begin{array}{ccc}
\mathrm{a}_{11}(\xi) & \mathrm{a}_{12}(\xi) \cdots & \mathrm{a}_{1 \mathrm{n}}(\xi) \\
\mathrm{a}_{21}(\xi) & \mathrm{a}_{22}(\xi) \cdots & \mathrm{a}_{2 \mathrm{n}}(\xi) \\
\vdots & \vdots & \vdots \\
\cdots & \mathrm{a}_{q s}(\xi) & \cdots \\
\vdots & \vdots & \vdots \\
\mathrm{a}_{\mathrm{n} 1}(\xi) & \mathrm{a}_{\mathrm{n} 2}(\xi) \cdots & \mathrm{a}_{\mathrm{nn}}(\xi)
\end{array}\right]\left[\begin{array}{c}
\chi_{1} \\
\chi_{2} \\
\vdots \\
\chi_{s} \\
\vdots \\
\chi_{\mathrm{n}}
\end{array}\right]=\left[\begin{array}{c}
0 \\
0 \\
\vdots \\
0 \\
\vdots \\
0
\end{array}\right]
$$

where 


$$
\mathrm{a}_{q s}(\xi)=K_{q s}\left\{\left(\xi_{s}^{2}\right)^{2}-\left(\xi^{2}\right)^{2}\left[1-\frac{\rho}{\left(\gamma_{q}^{(\xi)} \tan \gamma_{q}^{(\xi)}\right)}\right]\right\} .
$$

Hence values of $\xi 2$ can be obtained (iterated upon) which renders the determinant of matrix (20) equal to zero. Consequently for each of these values (roots) of $\xi 2$ the corresponding values of mode shape coefficients $\chi_{1}, \chi_{2}, \ldots \ldots \ldots \chi_{n}$., can be obtained. The determinant of this matrix equation is obtained by performing the $L U$ decomposition [17], whereupon the value of the determinant is the product of the diagonal terms. Subsequently these root values of $\xi 2$ which render the determinant zero are substituted back into equation (20) to obtain the corresponding values of the mode shape coefficients, $\chi_{s}$, (normalised to $\chi_{1}$ in the first instance and then to the largest value, ) which describe which structural modes are present and dominate.

The parameters which will give rise to conditions of strong structural/fluid vibration interaction will now be developed and postulated. Figure 2 shows a plot of a natural frequency, $\omega_{s}$, of the plate in vacuo and a natural frequency, $\omega_{m}$, of the acoustic cavity if the top plate was rigid. Both of these natural frequencies are plotted to a base of the controlling parameters, $L$; the depth of the cavity. Now, for any value of $s$, the natural frequency of the plate in vacuo is given by equation (5) and this value is independent of the depth $L$. A natural frequency of the solid bounded fluid cavity is obtained by now imposing the condition that

$$
\left.\frac{c}{L} \frac{\partial \phi}{\partial \bar{x}}\right|_{\bar{x}=1}=0 \quad \text { upon equation (12) }
$$

This results in $\gamma_{q}\left(\omega_{m}\right)=\frac{L}{a} \sqrt{\frac{\omega_{m}{ }^{2} a^{2}}{c^{2}}-\alpha_{q}{ }^{2}}=m \pi, m=1,2$ etc.

Note, in the special case(s) when $m=0$, this would imply only radial fluid modes with the fluid having zero axial component of velocity, this having no interaction with the axial vibration of the plate. Therefore, for $m \geq 1$,

$$
\begin{aligned}
\omega_{m} & =\frac{c}{a}\left[\left(\frac{m \pi a}{L}\right)^{2}+\alpha_{q}{ }^{2}\right]^{\frac{1}{2}} \\
\text { and } \quad \beta_{m} & =\frac{\omega_{m} L}{\pi c}=\sqrt{m^{2}+\frac{\bar{L}^{2}}{\pi^{2}} \alpha_{q}{ }^{2}}
\end{aligned}
$$

where $\bar{L}=\frac{L}{a}$

This is demonstrated in Figure 2. As $L$ increases all values of $\omega_{m}$ decrease and will, for appropriate values of $L=L_{c}$ correspond to values of $\omega_{S}$ of the plate in vacuo. In such circumstances there is strong structural/fluid vibration interaction characterised by a region of "veering" whence at $L=L_{c}$ the strongly interacting system will exhibit 
two natural frequencies close to each other, in which one will be structural/acoustic (st/ac) and the other acoustic/structural (ac/st).

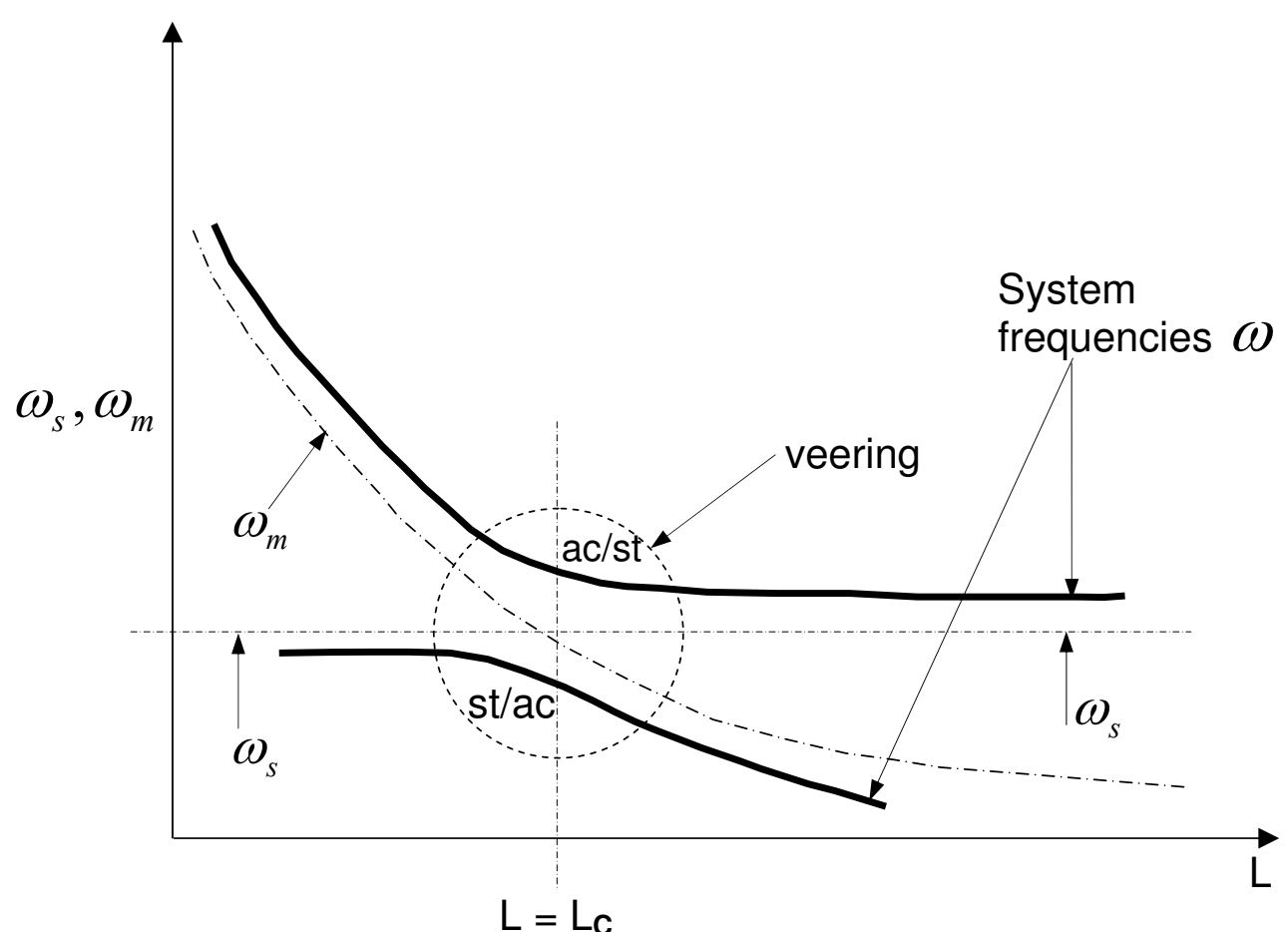

Figure 2 Illustration of strong structural / fluid vibration interaction

Therefore for $L=L_{c}$ equating equations (6) and (23) gives,

$$
\xi_{s}{ }^{2} \frac{h}{a^{2}} \sqrt{\frac{E}{12 \rho_{a}\left(1-\mu^{2}\right)}}=\frac{c}{a}\left[\left(\frac{m \pi a}{L_{c}}\right)^{2}+\alpha_{q}{ }^{2}\right]^{\frac{1}{2}}
$$

For the purpose of this study only the case where $q=1$, i.e. $\alpha_{q}=0$ (zero radial component of fluid velocity) will be studied, whence, for strong interaction, renders the condition.

$$
\bar{L}_{c}=\frac{L_{c}}{a}=\frac{m \pi c}{\xi_{s}^{2}} \sqrt{\frac{12 \rho_{d}\left(1-\mu^{2}\right)}{E}\left(\frac{a}{h}\right)^{2}}
$$


and $\quad \beta_{m}=\frac{\omega_{m} L}{\pi c}=m$

Prior to examining the characteristics of the strongly coupled vibration modes associated with various combinations of $s$ and $m$, the convergence of the analysis presented, and the sensitivity to small changes (errors) in the values of mode shapes associated with the free vibration of the plate in vacuo will be investigated.

For this exercise, and all subsequent results, the following parameters will be adopted; $c=343 \mathrm{~m} / \mathrm{s}$ (air), $\rho_{d}=7800 \mathrm{~kg} / \mathrm{m}^{3}, \mu=0.3, E=210 \mathrm{GN} / \mathrm{m}^{2}$ and $\left(\frac{a}{h}\right)=100$.

\section{Convergence}

For the convergence analysis consider a plate clamped around its perimeter. Furthermore the dimensions, $\bar{L}_{c}$ are selected, in accordance with equation (24), such that strong coupling exists between the first natural frequency of the plate in vacuo $(s=1)$ and the first natural frequency $(m=1)$ of the completely bounded cavity. For such a case the value of $\bar{L}_{c}$ is 6.7177 . Accordingly, Table 1 lists the values of $\xi^{2}$ and $\beta=\frac{\omega L}{\pi c}$ obtained for $n=2,4,6$ and 8 . In an attempt to identify particular coupled modes of vibration, Table 1 also lists the corresponding vector of mode shape coefficients, $\boldsymbol{q}_{\mathrm{s}}$. Furthermore the values of $\xi^{2}$ can be compared with those for the plate in vacuo; $\xi_{1}{ }^{2}=10.216, \xi_{2}{ }^{2}=39.771$ and $\xi_{3}{ }^{2}=89.104$ and the values of $\beta$ can be compared to values of $\beta_{m}=m$.

In this study, since in all cases we are dealing with some degree of structural/fluid vibration interaction, it would be erroneous to describe any mode of vibration as either purely a structural mode or an acoustic (fluid) mode. Rather reference will be made to the modes as either structural/acoustic (st/ac), to denote modes which are predominantly structural with acoustic interference, and likewise acoustic/structural (ac/st) to denote modes which are predominantly acoustic but with structural interference. 


\begin{tabular}{|c|c|c|c|c|}
\hline$n=2$ & $n=4$ & $n=6$ & $n=8$ & Comments \\
\hline $\begin{array}{l}9.6708,0.9467, \\
\left\{1,1.353 \times 10^{-2}\right\}\end{array}$ & $\begin{array}{l}9.6809,0.9476, \\
\left\{1,5.4 \times 10^{-3}--\right\}\end{array}$ & $\begin{array}{l}9.6815,0.9477, \\
\left\{1,5.2 \times 10^{-3}--\right\}\end{array}$ & $\begin{array}{l}9.6817,0.9477 \\
\left\{1,5.2 \times 10^{-3}--\right\}\end{array}$ & $\begin{array}{l}1^{\text {st }} \text { st } / \text { ac? } \\
(s=1, q=1, m=1), \\
\text { strong coupling } \\
\text { with } 1^{\text {st }} \text { ac/st }\end{array}$ \\
\hline $\begin{array}{l}10.763,1.0535 \\
\left\{1,-1.57 \times 10^{-2}\right\}\end{array}$ & $\begin{array}{l}10.754,1.0526 \\
\left\{1,-6.3 \times 10^{-3}--\right\}\end{array}$ & $\begin{array}{l}10.753,1.0526, \\
\left\{1,-6 \times 10^{-3}--\right\}\end{array}$ & $\begin{array}{l}\text { 10.753, 1.0526, } \\
\left\{1,-6 \times 10^{-3},--\right\}\end{array}$ & $\begin{array}{l}1^{\text {st }} \text { ac/st } ? \\
(s=1, q=1, m=1), \\
\text { strong coupling } \\
\text { with } 1^{\text {st }} \mathrm{st} / \mathrm{ac}\end{array}$ \\
\hline $\begin{array}{l}20.462,2.003, \\
\{1,-0.524\} \\
{[0.05,99.95]}\end{array}$ & $\begin{array}{l}20.466,2.0033, \\
\{1,-0.214,--\}\end{array}$ & $\begin{array}{l}20.466,2.0033, \\
\{1,-0.207,--\}\end{array}$ & $\begin{array}{l}20.466,2.0033, \\
\{1,-0.207,--\}\end{array}$ & $\begin{array}{l}2^{\mathrm{nd}} \mathrm{ac} / \mathrm{st} ? \\
(q=1, m=2)\end{array}$ \\
\hline $\begin{array}{l}30.646,2.999 \\
\{-0.39,1\} \\
{[0,100]}\end{array}$ & $\begin{array}{l}30.659,3.0011, \\
\{-0.967,1,--\}\end{array}$ & $\begin{array}{l}30.659,3.0011, \\
\{1,-1,---\}\end{array}$ & $\begin{array}{l}30.659,3.0011, \\
\{1,-1,---\}\end{array}$ & $\begin{array}{l}3^{\mathrm{rd}} \mathrm{ac} / \mathrm{st} ? \\
(q=1, m=3)\end{array}$ \\
\hline $\begin{array}{l}39.548,3.8712 \\
\left\{-3.7 \times 10^{-3}, 1\right\}\end{array}$ & $\begin{array}{l}39.648,3.8811, \\
\left\{-4 \times 10^{-3}, 1,--\right\}\end{array}$ & $\begin{array}{l}39.651,3.8813, \\
\left\{-4 \times 10^{-3}, 1,--\right\}\end{array}$ & $\begin{array}{l}39.651,3.8813, \\
\left\{-4 \times 10^{-3}, 1,--\right\}\end{array}$ & $\begin{array}{l}2^{\text {nd }} \text { st/ac ? } \\
(s=2, q=1, m=4)\end{array}$ \\
\hline $\begin{array}{l}41.064,4.02 \\
\left\{3.7 \times 10^{-2}, 1\right\}\end{array}$ & $\begin{array}{l}40.957,4.0092 \\
\left\{8.13 \times 10^{-2}, 1,--\right\}\end{array}$ & $\begin{array}{l}40.955,4.009 \\
\left\{8.38 \times 10^{-2}, 1,--\right\}\end{array}$ & $\begin{array}{l}40.954,4.0089 \\
\left\{8.42 \times 10^{-2}, 1,--\right.\end{array}$ & $\begin{array}{l}4^{\mathrm{th}} \mathrm{ac} / \mathrm{st} ? \\
(q=1, m=4)\end{array}$ \\
\hline
\end{tabular}

\section{$\underline{\text { Table } 1}$ Convergence $\quad \xi^{2}, \beta,\left\{\chi_{\mathrm{s}}\right\} \mathrm{T}$}

From Table 1, it is seen that convergence is extremely fast with respect to $n$; requiring only $n=6$ for a fully converged result for these lower modes. Accordingly, forthwith $n=6$ will be used throughout.

\section{$\underline{\text { Sensitivity to small changes in eigenvectors }}$}

With reference to equation (17), it can be seen that two main influencing factors are the natural frequencies of the plate in vacuo $\left(\omega_{s}\right)$ and the associated mode shapes contained in $K_{q s}$ (equation (15)). For simple structural elements such as that considered in this study accurate descriptions of these modal parameters can easily be obtained from standard exact analysis. However in more complex structures often the natural frequencies $\left(\omega_{s}\right)$ are obtained from energy/numerical techniques, e.g. Rayleigh-Ritz, finite element etc. However with modern high performance computers, the accuracy of the natural frequencies can correspond exceedingly close to the exact values. However in the case of the associated mode shapes, $\psi_{S}(\bar{r})$, accurate equations describing these are difficult to obtain using energy methods (where an approximate form of mode shape is assumed) and finite element procedures (where mode shapes are produced in the form of discrete numbers produced from a numerical treatise of the problem). Therefore, the effect of changes in mode shape upon the sensitivity of the results produced by the coupled system equation (20) will 
be investigated. For this, once again consider the case of a plate clamped around the boundaries and, as before, the dimensions are set such that there is strong coupling between the first natural frequency of the plate in vacuo and the first acoustic natural frequency of the solid bounded cavity $(s=1, m=1)$.

Table 2 shows results corresponding to the first six natural modes of the coupled system. In this case, however, the same natural frequencies of the clamped plate in vacuo was used, but, instead of using the corresponding plate natural mode shape function for a clamped plate that corresponding to a simply supported plate in the formulation of $K_{q s}$ was used. This represents a crude form of approximation of the actual and correct mode shape of a clamped plate. Accordingly, Table 2 list the details of these six natural modes computed using these erroneous normal mode functions. For direct comparison, Table 2 lists the corresponding (correct) values in which both the natural frequencies and associated mode shapes for a plate clamped around the periphery are used in equation (20-21) (values contained in square, [ ], brackets). The contents of Table 2 indicate a remarkable insensitivity of values and vectors describing the natural modes of the coupled system to errors in the natural mode functions of the plate in vacuo. In other words, it would appear that the determinant of the system matrix equation (20-21) is very insensitive to relatively significant changes in the values of $K_{q s .}$. This is an interesting observation as it indicates that the method can be used with a good degree of confidence if one can only present equation (20-21) with approximate mode shapes, which is often the case in practice.

\begin{tabular}{|l|lll|}
\hline$\xi^{2}, \beta$ & & \\
\hline $\begin{array}{l}9.6011,0.9398 \\
{[9.6815,0.9477]}\end{array}$ & $\left\{\chi_{\mathrm{s}}\right\}^{\mathrm{T}}=\{1, \sim 0, \sim 0,--------\}$ & {$[\{1, \sim 0, \sim 0,--------\}]$} \\
\hline $\begin{array}{l}10.843,1.0613 \\
{[10.753,1.0526]}\end{array}$ & $\left\{\chi_{\mathrm{s}}\right\}^{\mathrm{T}}=\{1, \sim 0, \sim 0,------\}$ & {$[\{1, \sim 0, \sim 0,-\cdots----\}]$} \\
\hline $\begin{array}{l}20.048,2.0047 \\
{[20.466,2.0033]}\end{array}$ & $\left\{\chi_{\mathrm{s}}\right\}^{\mathrm{T}}=\{1,-0.185, \sim 0,---\}$ & {$[\{1,-0.207, \sim 0,---\}]$} \\
\hline $\begin{array}{l}30.067,3.0021 \\
{[30.659,3.0011]}\end{array}$ & $\left\{\chi_{\mathrm{s}}\right\}^{\mathrm{T}}=\{1,-0.9, \sim 0-----\}$ & {$[\{1,-1, \sim 0, \sim 0,--\}]$} \\
\hline $\begin{array}{l}39.654,3.8816 \\
{[39.651,3.8813]}\end{array}$ & $\left\{\chi_{\mathrm{s}}\right\}^{\mathrm{T}}=\{\sim 0,1, \sim 0, \sim 0,--\}$ & {$[\{\sim 0,1, \sim 0, \sim 0,--\}]$} \\
\hline $\begin{array}{l}40.094,4.0076 \\
{[40.955,4.0089]}\end{array}$ & $\left\{\chi_{\mathrm{s}}\right\}^{\mathrm{T}}=\{\sim 0,1, \sim 0,------\}$ & {$[\{\sim 0,1, \sim 0,------\}]$} \\
\hline
\end{tabular}

Table 2 Comparison of results $\left(\xi^{2},\left\{\chi_{\mathrm{s}}\right\}^{\mathrm{T}}\right)$ of using two different mode shapes in formulation of $K_{q s .}$.

The results presented in Table 2 demonstrate that the equation (20-21) renders roots which are relatively insensitive to the form of the mode shape used to describe the vibration of the plate in vacuo.

\section{SUB-SYSTEM MODAL ENERGY.}

In Table 1 reference was made to st/ac and ac/st mode to indicate whether the mode of vibration was predominantly a structural or acoustic respectively. The modes are labelled according to; 
1. the proximity of $\xi^{2}$ to $\xi_{\mathrm{s}}^{2}$

2. the proximity of $\beta$ to $\beta \mathrm{m}$, and

3. the form of the vector of mode shape coefficients $\left\{\chi_{\mathrm{s}}\right\}$.

In general this is an inaccurate and unsatisfactory means of trying to describe these couple modes. For example, if one considers the $3^{\text {rd }}$ and $4^{\text {th }}$ row of Table 1 for $n=6$, the values of $\xi^{2}$ (20.466 and 30.659 respectively) are well removed from $\xi_{1}^{2}(10.216)$ and $\xi_{2}^{2}$ (39.771) and the corresponding values of $\beta$ are exceedingly close to 2 and 3 . This indicates a strong ac/st mode. However, the issue is clouded as it can be seen that for each of those two modes, the mode shape of the plate will be a combination of the first and second natural mode shapes of the plate in vacuo.

Accordingly, in an attempt to describe such modes in a clearer and more quantifiable manner, attention will now be focussed on the kinetic energy associated with coupled structural/fluid vibration interacting systems.

For the plate the kinetic energy, $K E_{p}$, is calculated from;

$$
K E_{p}=\pi \rho_{d} h a^{4} \omega^{2} \int_{0}^{1}\left[\sum_{s=1}^{n} \chi_{s} \psi_{s}(\bar{r})\right]^{2} \bar{r} d \bar{r}
$$

and since the eigenvectors, $\psi_{S}(\bar{r})$, are orthogonal, then,

$$
K E_{p}=\sum_{s=1}^{n} K E_{p s}
$$

where $K E_{p s}=\pi \rho_{d} h a^{4} \omega^{2} \int_{0}^{1}\left[\chi_{s} \psi_{s}(\bar{r})\right]^{2} \bar{r} d \bar{r}$

For the fluid the kinetic energy, $K E_{f}$, is calculated from;

$$
K E_{f}=\rho_{f} \pi a^{2} L \int_{00}^{1} \int_{0}^{1}\left[\left(V_{\bar{r}}\right)^{2}+\left(V_{\bar{x}}\right)^{2}\right] \bar{r} d \bar{r} d \bar{x}
$$

where $V_{r}=c \frac{\partial \bar{\phi}}{\partial \bar{r}} ; \quad V_{\bar{x}}=\frac{a c}{L} \frac{\partial \bar{\phi}}{\partial \bar{x}}$

where $\bar{\phi}=\sum_{q=1}^{\infty} B_{q} \cos \left(\gamma_{q}^{(\omega)} \bar{x}\right) J_{0}\left(\alpha_{q} \bar{r}\right) e^{i \omega t}$

Therefore $\quad V_{r}=\sum_{q=1}^{n} V_{r q}$ and $V_{x}=\sum_{q=1}^{n} V_{x q}$

Once again, because of orthogonality of the eigenvectors describing $\bar{\phi}$ 


$$
K E_{f}=\sum_{q=1}^{n} K E_{f q}^{r}+\sum_{q=1}^{n} K E_{f q}^{x}
$$

where $K E_{f q}^{r}=\rho_{f} \pi a^{2} L \int_{00}^{1} \int_{0}^{1}\left(V_{\bar{r} q}\right)^{2} \bar{r} d \bar{r} d \bar{x}$

and $K E_{f q}^{x}=\rho_{f} \pi a^{2} L \int_{00}^{1} \int_{0}^{1}\left(V_{\bar{x} q}\right)^{2} \bar{r} d \bar{r} d \bar{x}$

and both $V_{r q}$ and $V_{x q}$ are now obtained from equations (12) and (14). Also, the percentage energy associated with $K E_{f}$ and $K E_{p}$ are expressed as,

$\% K E_{f}^{x}=\frac{\sum_{q=1}^{n} K E_{f q}^{x}}{\left(K E_{f}+K E_{p}\right)} \times 100 \% \quad$ for total axial fluid energy

$\% K E_{f}^{r}=\frac{\sum_{q=1}^{n} K E_{f q}^{r}}{\left(K E_{f}+K E_{p}\right)} \times 100 \% \quad$ for total radial fluid energy

and $\% K E_{p}=\frac{K E_{p}}{\left(K E_{f}+K E_{p}\right)} \times 100 \% \quad$ for total plate energy

Also a set of eigenvectors for the plate and fluid is

$\left\{\% \boldsymbol{K E}_{f q}^{r}\right\}=\left\{\% K E_{f 1}^{r}, \% K E_{f 2}^{r},---\% K E_{f q}^{r},---\% K E_{f n}^{r}\right\}=$ eigenvector of radial kinetic energy components of fluid

$\left\{\% \boldsymbol{K} \boldsymbol{E}_{f q}^{x}\right\}=\left\{\% K E_{f 1}^{x}, \% K E_{f 2}^{x},---\% K E_{f q}^{x},---\% K E_{f n}^{x}\right\}=$ eigenvector of axial kinetic energy components of fluid, and,

$\left\{\% \boldsymbol{K} \boldsymbol{E}_{p s}\right\}=\left\{\% K E_{p 1}, \quad \% K E_{p 2},---\% K E_{p s},---\% K E_{p n}\right\}=$ eigenvector of kinetic energy of lateral vibration components of the plate

where $\% K E_{f q}^{r}=\frac{K E_{f q}^{r}}{\left[K E_{f}+K E_{p}\right]} \times 100 \%$

$$
\% K E_{f q}^{x}=\frac{K E_{f q}^{x}}{\left[K E_{f}+K E_{p}\right]} \quad \mathbf{x} \quad 100 \%
$$

and $\% K E_{p s}=\frac{K E_{p s}}{\left[K E_{f}+K E_{p}\right]} \times 100 \%$ 
Consequently, using the above relative percentage energies, the characteristics of a circular clamped disc in strong interaction with an acoustic cavity as described are investigated. Consider the case where $\bar{L}_{c}$ is 6.7177 which, as before, results in a condition of strong coupling between the first mode of the plate in vacuo $(s=1)$, and the first $(m=1)$ axial mode of the fluid cavity if the plate is assumed rigid. In all cases the ratio of $\frac{a}{h}=100$ and we will only consider roots of the system matrix equation (20-21), $\xi^{2}$, up to those close to that corresponding to the third of the plate in vacuo, $\xi_{3}^{2}$, equal to 89.104 . Accordingly Table 3 list the frequency roots, $\xi^{2}$, together with the associated vectors; $\left\{\chi_{s}\right\}^{\mathrm{T},}\left\{\% \boldsymbol{K} \boldsymbol{E}_{p s}\right\},\left\{\% \boldsymbol{K E}_{f q}^{x}\right\}$ and $\left\{\% \boldsymbol{K E}_{f q}^{r}\right\}$. 


\begin{tabular}{|c|c|c|c|}
\hline $9.6815,0.9477$ & $\begin{array}{l}\left\{\chi_{\mathrm{s}}\right\}^{\mathrm{T}} \\
\left\{\% \boldsymbol{K} \boldsymbol{E}_{p s}\right\} \\
\left\{\% \boldsymbol{K} \boldsymbol{E}_{f q}^{x}\right\} \\
\left\{\% \boldsymbol{K} \boldsymbol{E}_{f q}^{r}\right\}\end{array}$ & $\begin{array}{l}=\{1, \sim 0, \sim 0,--------\} \\
=\{47.88, \sim 0, \sim 0,---\} \\
=\{52.03, \sim 0, \sim 0,---\} \\
=\{\sim 0, \sim 0,-------\}\end{array}$ & $\begin{array}{l}\text { Strongly coupled st/ac or ac/st } \\
\text { mode at } s=1, q=1, m \sim 1 .\end{array}$ \\
\hline $10.753,1.0526$ & $\begin{array}{l}\left\{\chi_{\mathrm{s}}\right\}^{\mathrm{T}} \\
\left\{\% \boldsymbol{K} \boldsymbol{E}_{p s}\right\} \\
\left\{\% \boldsymbol{K} \boldsymbol{E}_{f q}^{x}\right\} \\
\left\{\% \boldsymbol{K} \boldsymbol{E}_{f q}^{r}\right\}\end{array}$ & $\begin{array}{l}=\{1, \sim 0, \sim 0,------\} \\
=\{50.99, \sim 0, \sim 0,--\} \\
=\{48.91, \sim 0, \sim 0,--\} \\
=\{\sim 0, \sim 0, \sim 0,------\}\end{array}$ & $\begin{array}{l}\text { Coupled ac/st mode at } s=1, q=1, \\
m \sim 1 .\end{array}$ \\
\hline $20.466,2.0033$ & $\begin{array}{l}\left\{\mathcal{X}_{\mathbf{s}}\right\}^{\mathrm{T}} \\
\left\{\% \boldsymbol{K} \boldsymbol{E}_{p s}\right\} \\
\left\{\% \boldsymbol{K} \boldsymbol{E}_{f q}^{x}\right\} \\
\left\{\% \boldsymbol{K} \boldsymbol{E}_{f q}^{r}\right\}\end{array}$ & $\begin{array}{l}=\{1,-0.207, \sim 0,---\} \\
=\{0.5, \sim 0, \sim 0,-----\} \\
=\{99.5, \sim 0, \sim 0,--\} \\
=\{\sim 0, \sim 0, \sim 0,-----\}\end{array}$ & $\begin{array}{l}\text { Weakly coupled ac/st mode. } \\
\text { Almost total fluid axial energy at } \\
q=1, m \sim 2 .\end{array}$ \\
\hline $30.659,3.0011$ & $\begin{array}{l}\left\{\mathcal{X}_{\mathrm{s}}\right\}^{\mathrm{T}} \\
\left\{\% \boldsymbol{K} \boldsymbol{E}_{p s}\right\} \\
\left\{\% \boldsymbol{K} \boldsymbol{E}_{f q}^{x}\right\} \\
\left\{\% \boldsymbol{K} \boldsymbol{E}_{f q}^{r}\right\}\end{array}$ & $\begin{array}{l}=\{1,-1, \sim 0, \sim 0,--\} \\
=\{\sim 0, \sim 0, \sim 0,----\} \\
=\{99.99, \sim 0, \sim 0,--\} \\
=\{\sim 0, \sim 0, \sim 0,-----\}\end{array}$ & $\begin{array}{l}\text { Weakly coupled ac/st mode. } \\
\text { Almost total fluid axial energy at } \\
q=1, m \sim 3 \text {. }\end{array}$ \\
\hline $39.651,3.8813$ & $\begin{array}{l}\left\{\chi_{\mathbf{s}}\right\}^{\mathrm{T}} \\
\left\{\% \boldsymbol{K} \boldsymbol{E}_{p s}\right\} \\
\left\{\% \boldsymbol{K} \boldsymbol{E}_{f q}^{x}\right\} \\
\left\{\% \boldsymbol{K} \boldsymbol{E}_{f q}^{r}\right\}\end{array}$ & $\begin{array}{l}=\{\sim 0,1, \sim 0, \sim 0,--\} \\
=\{\sim 0,93.53, \sim 0,-\} \\
=\{6.22, \sim 0, \sim 0,-\} \\
=\{\sim 0,1.43, \sim 0,---\}\end{array}$ & $\begin{array}{l}\text { Coupled st/ac mode at } s=2, q=1, \\
m \sim 4 .\end{array}$ \\
\hline $40.955,4.0089$ & $\begin{array}{l}\left\{\mathcal{X}_{\mathbf{s}}\right\}^{\mathrm{T}} \\
\left\{\% \boldsymbol{K} \boldsymbol{E}_{p s}\right\} \\
\left\{\% \boldsymbol{K} \boldsymbol{E}_{f q}^{x}\right\} \\
\left\{\% \boldsymbol{K} \boldsymbol{E}_{f q}^{r}\right\}\end{array}$ & $\begin{array}{l}=\{\sim 0,1, \sim 0,------\} \\
=\{\sim 0,6.01, \sim 0,--\} \\
=\{93.90, \sim 0,-----\} \\
=\{\sim 0, \sim 0, \sim 0,----\}\end{array}$ & $\begin{array}{l}\text { Weakly coupled ac/st mode. } \\
\text { Almost total fluid axial energy at } \\
q=1, m \sim 4 \text {. }\end{array}$ \\
\hline $51.099,5.002$ & $\begin{array}{l}\left\{\mathcal{X}_{\mathrm{s}}\right\}^{\mathrm{T}} \\
\left\{\% \boldsymbol{K} \boldsymbol{E}_{p s}\right\} \\
\left\{\% \boldsymbol{K} \boldsymbol{E}_{f q}^{x}\right\} \\
\left\{\% \boldsymbol{K} \boldsymbol{E}_{f q}^{r}\right\}\end{array}$ & $\begin{array}{l}=\{0.54,1,-0.17,--\} \\
=\{\sim 0, \sim 0, \sim 0,----\} \\
=\{99.9, \sim 0,------\} \\
=\{\sim 0, \sim 0, \sim 0,----\}\end{array}$ & $\begin{array}{l}\text { Weakly coupled ac/st mode. } \\
\text { Almost total fluid axial energy at } \\
q=1, m \sim 5 \text {. }\end{array}$ \\
\hline $61.3,6.001$ & $\begin{array}{l}\left\{\mathcal{X}_{\mathrm{s}}\right\}^{\mathrm{T}} \\
\left\{\% \boldsymbol{K} \boldsymbol{E}_{p s}\right\} \\
\left\{\% \boldsymbol{K} \boldsymbol{E}_{f q}^{x}\right\} \\
\left\{\% \boldsymbol{K} \boldsymbol{E}_{f q}^{r}\right\}\end{array}$ & $\begin{array}{l}=\{0.78,1,-0.46,--\} \\
=\{\sim 0, \sim 0, \sim 0,----\} \\
=\{99.99, \sim 0, \sim 0,--\} \\
=\{\sim 0, \sim 0, \sim 0,-----\}\end{array}$ & $\begin{array}{l}\text { Weakly coupled ac/st mode. } \\
\text { Almost total fluid axial energy at } \\
q=1, m \sim 6 .\end{array}$ \\
\hline
\end{tabular}




\begin{tabular}{|c|c|c|}
\hline$\xi^{2}, \beta$ & & Mode Description \\
\hline $71.52,7.0009$ & $\begin{array}{l}\left\{\boldsymbol{\chi}_{\mathrm{s}}\right\}^{\mathrm{T}}=\{0.83,0.9,-1,---\} \\
\left\{\% \boldsymbol{K}_{p s}\right\}=\{\sim 0, \sim 0, \sim 0,-----\} \\
\left\{\% \boldsymbol{K}_{f q}^{x}\right\}=\{99.99, \sim 0,------\} \\
\left\{\% \boldsymbol{K E}_{f q}^{r}\right\}=\{\sim 0, \sim 0, \sim 0,------\}\end{array}$ & $\begin{array}{l}\text { Weakly coupled ac/st mode. } \\
\text { Almost all fluid axial energy at } \\
q=1, m \sim 7 .\end{array}$ \\
\hline $81.73,8.0004$ & $\begin{array}{l}\left\{\boldsymbol{\chi}_{\mathrm{s}}\right\}^{\mathrm{T}}=\{0.28,0.28,-1,--\} \\
\left\{\% \boldsymbol{K} \boldsymbol{E}_{p s}\right\}=\{\sim 0, \sim 0, \sim 0,--- \\
\left\{\% \boldsymbol{K}_{f}^{x}\right\}=\{99.99, \sim 0,-----\} \\
\left\{\% \boldsymbol{K} \boldsymbol{E}_{f q}^{r}\right\}=\{\sim 0, \sim 0, \sim 0,----\}\end{array}$ & $\begin{array}{l}\text { Weakly coupled ac/st mode. } \\
\text { Almost all fluid axial energy at } \\
q=1, m \sim 8 .\end{array}$ \\
\hline $83.7,8.193$ & $\begin{array}{l}\left\{\boldsymbol{\chi}_{\mathrm{s}}\right\}^{\mathrm{T}}=\{0.18,-0.22,1,---\} \\
\left\{\% \boldsymbol{K}_{p s}\right\}=\{\sim 0, \sim 0, \sim 0,-----\} \\
\left\{\% \boldsymbol{K E}_{f q}^{x}\right\}=\{\sim 0, \sim 0, \sim 0,----\} \\
\left\{\% \boldsymbol{K E}_{f q}^{r}\right\}=\{\sim 0,99.99, \sim 0,---\}\end{array}$ & $\begin{array}{l}\text { Weakly coupled ac/st mode. } \\
\text { Almost all fluid radial energy at } \\
q=2 \text {. }\end{array}$ \\
\hline $84.32,8.254$ & $\begin{array}{l}\left\{\chi_{\mathrm{s}}\right\}^{\mathrm{T}}=\{0.16,-0.19,1,---\} \\
\left\{\% \boldsymbol{K} \boldsymbol{E}_{p s}\right\}=\{\sim 0, \sim 0, \sim 0,------\} \\
\left\{\% \boldsymbol{K E}_{f q}^{x}\right\}=\{\sim 0,1.47, \sim 0,----\} \\
\left\{\% \boldsymbol{K E}_{f q}^{r}\right\}=\{\sim 0,98.5, \sim 0,----\}\end{array}$ & $\begin{array}{l}\text { Same as above except small axial } \\
\text { fluid component at } q=2, m \sim 8 \text {. }\end{array}$ \\
\hline $86.15,8.433$ & $\begin{array}{l}\left\{\boldsymbol{\chi}_{\mathrm{s}}\right\}^{\mathrm{T}}=\{\sim 0,-0.1,1,-----\} \\
\left\{\% \boldsymbol{K}_{p s}\right\}=\{\sim 0, \sim 0, \sim 0,----\} \\
\left\{\% \boldsymbol{K}_{\text {甚 }}^{x}\right\}=\{\sim 0,5.62, \sim 0,---\} \\
\left\{\% \boldsymbol{K} \boldsymbol{E}_{f q}^{r}\right\}=\{\sim 0,94.38, \sim 0,--\}\end{array}$ & $\begin{array}{l}\text { Same as above except growing } \\
\text { axial fluid component. }\end{array}$ \\
\hline $88.85,8.698$ & $\begin{array}{l}\left\{\chi_{\mathrm{s}}\right\}^{\mathrm{T}}=\{\sim 0, \sim 0,1, \sim 0,--\} \\
\left\{\% \boldsymbol{K}_{p s}\right\}=\{\sim 0, \sim 0,63,----\} \\
\left\{\% \boldsymbol{K E}^{x}{ }_{f q}\right\}=\{0.4,4.3, \sim 0,---\} \\
\left\{\% \boldsymbol{K E}_{f q}^{r}\right\}=\{\sim 0,32, \sim 0,--\}\end{array}$ & $\begin{array}{l}\text { Strongly coupled st/ac mode } \\
\text { between } \mathrm{s}=3 \text { on plate and radial } \\
\text { fluid mode at } q=2 .\end{array}$ \\
\hline $89.31,8.742$ & $\begin{array}{l}\left\{\mathcal{X}_{\mathrm{s}}\right\}^{\mathrm{T}}=\{\sim 0, \sim 0,1, \sim 0,--\} \\
\left\{\% \boldsymbol{K} \boldsymbol{E}_{p s}\right\}=\{\sim 0, \sim 0,36.9,---\} \\
\left\{\% \boldsymbol{K} \boldsymbol{E}_{f q}^{x}\right\}=\{1,7.4, \sim 0,-----\} \\
\left\{\% \boldsymbol{K} \boldsymbol{E}_{f q}^{r}\right\}=\{\sim 0,55.3, \sim 0,----\}\end{array}$ & $\begin{array}{l}\text { Strongly coupled ac/st mode } \\
\text { between } \mathrm{s}=3 \text { on plate and radial } \\
\text { fluid mode at } q=2 .\end{array}$ \\
\hline $91.97,9.002$ & $\begin{array}{l}\left\{\chi_{\mathrm{s}}\right\}^{\mathrm{T}}=\{\sim 0, \sim 0,1, \sim 0,--\} \\
\left\{\% \boldsymbol{K}_{p s}\right\}=\{\sim 0, \sim 0,0.6,----\} \\
\left\{\% \boldsymbol{K E}_{f q}^{x}\right\}=\{99.4, \sim 0,-----\} \\
\left\{\% \boldsymbol{K} \boldsymbol{E}_{f q}^{r}\right\}=\{\sim 0, \sim 0, \sim 0,-----\}\end{array}$ & $\begin{array}{l}\text { Weakly coupled ac/st mode. } \\
\text { Almost all fluid axial energy at } \\
q=1, m \sim 9 .\end{array}$ \\
\hline
\end{tabular}

Table 3 - Modes of free vibration of structural/fluid interacting system with associated energy vectors.

From the results of Table 3 one has a clear impression of the physical significance and characteristics of each mode of vibration. For example, consider now once again the two modes at values of $\xi^{2}$ of 20.466 and 30.659 respectively. From Table 3 we can immediately see that these are two weakly coupled, strong acoustic modes and as such the associated vectors, $\left\{\chi_{s}\right\}^{\mathrm{T}}$, are unrepresentative of the actual situation. $\mathrm{A}$ interesting feature illustrated in Table 3 is that for the higher modes of vibration around the frequencies close to that of the natural frequency associated with the plate in vacuo at $s=3, \xi_{3}^{2}$ (89.104), (see the cases $\xi^{2}=88.85$ and 89.31), the modes have a strong acoustic component on the basis of percentage energy; $37 \%$ and $63 \%$ respectively. This indicates that at these higher natural frequencies of the coupled 
system, the fluid behaves as an energy absorber thus protecting the structure from the high vibratory energy associated with the plate in vacuo at the third natural mode. Too illustrate this point, Figure 3a shows a typical vibration energy plot (to the base of non-dimensional frequency) which one would expect for this plate in vacuo if damping was negligible. On the other hand Figure $3 \mathrm{~b}$ shows the corresponding plot for the vibration energy of the plate if it was now in contact with the solid cylindrical fluid cavity described above and only the vibration on the surface of the plate is measured; as is the case in standard modal analysis. One can immediately see the dramatic difference between the two plots and illustrates well the implications of structural/fluid interaction. Such a situation can obviously give rise to difficulties for dynamicists performing standard procedures of structural modal analysis.

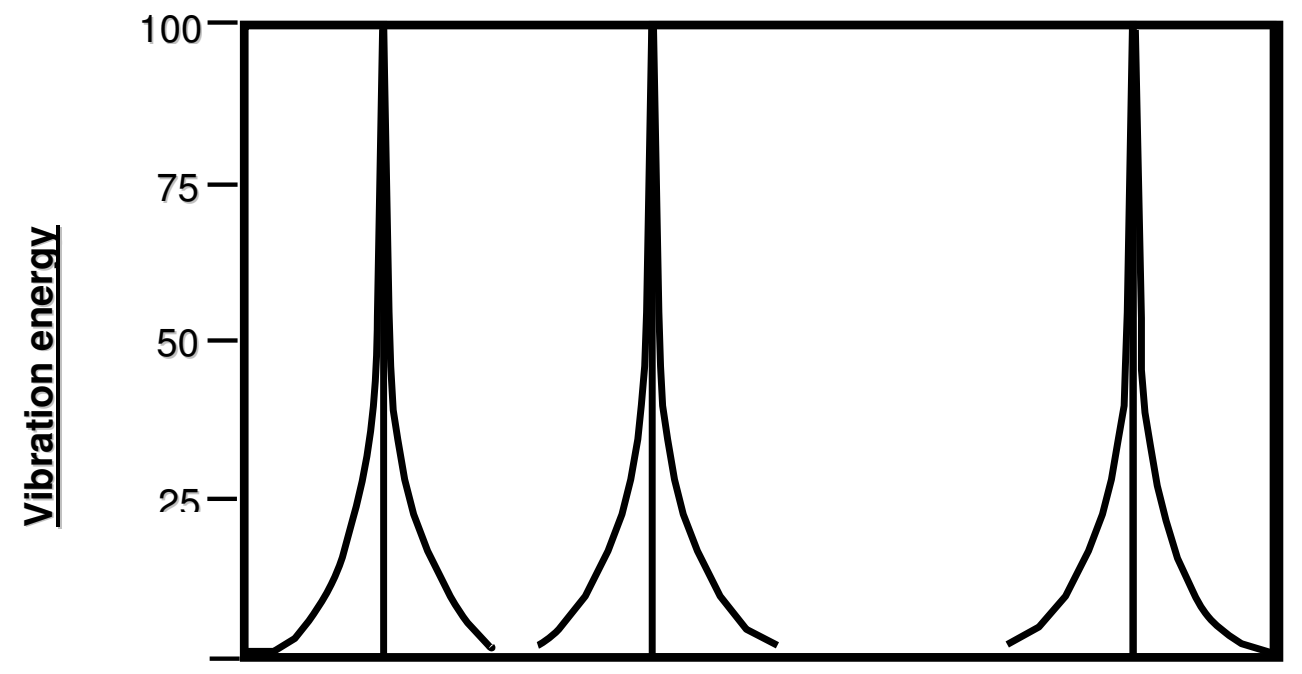


0
10.2
39.8
89.1

Figure 3a Frequency response of plate in vacuo

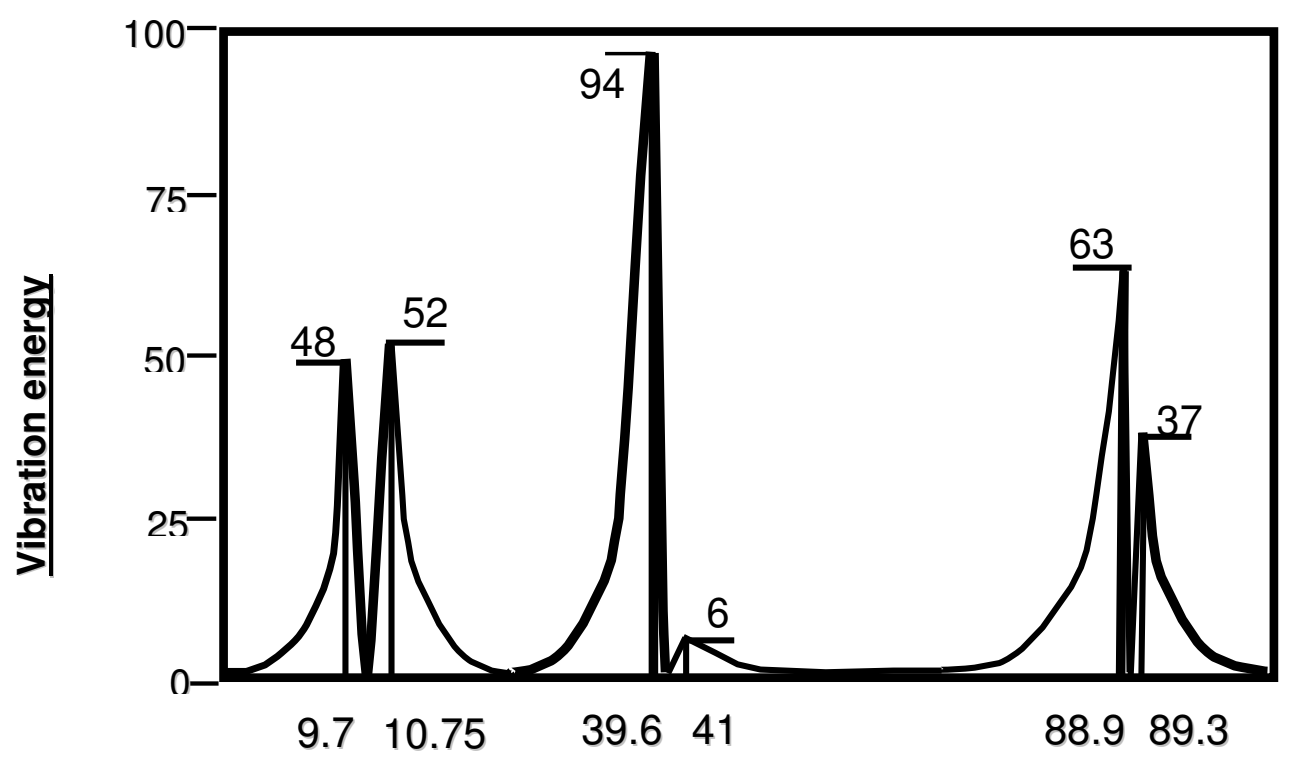

Figure $3 b$ Frequency response of plate in interaction

\section{CONCLUSIONS}

A theoretical - analytical method, based on the based upon the Euler Bernoulli and Helmholtz equations and the Galerkin method, has been developed for the frequencymodal analysis of a coupled vibroacoustic system. This form of analysis results in an elegant system eigen matrix equation which readily indicated the relevant parameters governing the behaviour of the coupled system and could be equally applied to other more general forms of plates and structure. The convergence of the solution is fast and would appear to be very insensitive to changes of the assumed form of mode shapes associated with the plate in vacuo. The analysis was extended to describe the natural coupled modes in terms of the relative energy associated with each of the two sub- 
systems, plate and fluid. This was demonstrated to give an excellent insight into the physical characteristics of structural/fluid coupled modes and in a forced vibration situation, the analyst would gain valuable information with respect to where the energy of excitation would direct itself. In addition, by considering the general characteristics of the coupled system shown by the sub-system modal energy representation, it can be deduced that general structural modal analysis for the purpose of predicting in service vibration response and the non destructive method of structural health monitoring based upon vibration signatures would require to be deployed with increasing care when applied to such coupled systems.

\section{REFERENCES}

[1] Dowell, E.H., Gorman, G.F. and Smith, D.A.: Acoustoelasticity: General theory, acoustic natural modes and forced response to sinusoidal excitation, including comparisons with experiment. J. Sound Vib., 1977, 52(4), 519-542.

[2] Fahy, F.: Sound and Structural Vibration. Academic Press Limited, London, 1993.

[3] Markuš, Š., Nanasi, T., and Šimková, O.: Vibroacoustics of enclosed cavities. in: Dynamics of Bodies in Interaction with Surroundings (ed. Guz A.N.), Naukova dumka, Kijev, 1991 (in Russian).

[4] Pretlove, J.: Free Vibrations of a Rectangular Panel Backed by a closed Rectangular Cavity. J. Sound Vib., 1965, 2 (3), 197-209.

[5] Pan, J. and Bies, D.A.: The effect of fluid-structural coupling on sound waves in an enclosure-Experimental part. J. Acoust. Soc. Am, 1990, 87(2), 708-721.

[6] Bokil, V.B. and Shirahatti, U.S.: A technique for the modal analysis of soundstructure interaction problems. J. Sound Vib., 1994, 173 (1), 23-41.

[7] Rajalingham, C., Bhat, R.B. and Xistris, G. D.: Vibration of circular membrane backed by cylindrical cavity. Int. J. Mech. Sci.. 1998, 40( 8), 723-734.

[8] Lee, M.-R. and Singh, R.: Analytical formulations for annular disk sound radiation using structural modes. J. Acoust. Soc. Am. 1994, 95(6), 3311-3313.

[9] Gorman D G, Reese J M, Horacek J and Dedouch K: Vibration analysis of a circular disc backed by a cylindrical cavity. Proceedings of the Institution of Mechanical Engineers, Part C, 2001, 215, pp1303-1311.

[10] D, G, Gorman, C. K. Lee, I. A. Craighead and J. Horacek: Transverse vibration analysis of a prestressed thin circular plate in contact with an acoustic cavity. Engineering Mechanics - Association for Engineering Mechanics, Engineering Academy of the Czech Republic, 2005, 12 (6), pp 417-427. 
[11] Bauer, H.F. and Chiba, M.: Hydroelastic viscous oscillations in a circular cylindrical container with an elastic cover. J. Fluids Struct., 2000, 14, pp 917-936.

[12] Amabili, M., Frosali G. and Kwak, M. K. : Free vibrations of annular plates coupled with fluids. Journal of Sound and Vibration, 1996, 191(5), pp. 825-846.

[13] Amabili, M.: Effect of finite fluid depth on the hydroelastic vibrations of circular and annular plates. Journal of Sound and Vibration, 1996, 193(4), pp. 909-925.

[14] Gorman, D.G, Lee, C. K, Reese, J.M and Horacek, J: Vibration analysis of a thin circular plate influenced by liquid/gas interaction in a cylindrical cavity. Journal of Sound and Vibration, 2005, 279, pp601-618.

[15] Leissa, A.W.: Vibration of Plates. NASA SP-160, Washington, 1969.

[16] McLachlan, N.W.: Bessel Functions for Engineers. Oxford Engineering Science Series, 1948,(Oxford University Press, London).

[17] Press, W.H, Flannery, B.P., Teukolsky, S.A., and Vetterling, W.T.: Numerical Recipes, Cambridge University Press, 1988, pp31-39.

This research was supported by the Royal Society (London) and the Grant Agency of the Academy of Sciences of the Czech Republic by the project A2076101/01 Natural vibration and stability of shells in interaction with fluid. 